\title{
ON THE CANONICAL REAL STRUCTURE ON WONDERFUL VARIETIES
}

\author{
D. AKHIEZER AND S. CUPIT-FOUTOU
}

\begin{abstract}
We study equivariant real structures on spherical varieties. We call such a structure canonical if it is equivariant with respect to the involution defining the split real form of the acting reductive group $G$. We prove the existence and uniqueness of a canonical structure for homogeneous spherical varieties $G / H$ with $H$ self-normalizing and for their wonderful embeddings. For a strict wonderful variety we give an estimate of the number of real form orbits on the set of real points.
\end{abstract}

\section{Contents}

Introduction

1. Wonderful varieties

2. Finiteness theorem

3. General properties of equivariant real structures

4. The canonical real structure

5. Real part: local structure and $G_{0}^{\sigma}$-orbits

Appendix A. Spherical varieties: invariants and local structure 14 A.1. Luna-Vust invariants of spherical homogeneous spaces 14

A.2. Local structure References 16

\section{INTRODUCTION}

A real structure on a complex manifold $X$ is an anti-holomorphic involution $\mu: X \rightarrow X$. The set of fixed points $X^{\mu}$ of $\mu$ is called the real part of $(X, \mu)$. If it is clear from the context which $\mu$ is considered then the real part will be denoted by $\mathbb{R} X$. In our paper, we are interested in the algebraic case. This means that $X$ is a complex algebraic variety, which we will assume non-singular though this is not needed for the

Supported by SFB/TR 12, Symmetry and universality in mesoscopic systems, of the Deutsche Forschungsgemeinschaft. 
definition of a real structure. Also, $\mu$ is algebraic in the sense that for any function $f$ regular at $x \in X$ the function $\overline{f \circ \mu}$ is regular at $\mu(x)$.

It is not easy to classify all real structures on a given variety $X$. Much work is done for compact toric varieties, where one has the notion of a toric real structure. Namely, if $X$ is a toric variety acted on by an algebraic torus $T$ then a real structure $\mu: X \rightarrow X$ is said to be toric if $\mu$ normalizes the $T$-action. It is natural to classify toric real structures up to conjugation by toric automorphisms, i.e., by automorphisms of $X$ normalizing the $T$-action. Again, such a classification is not easy. For toric surfaces and threefolds it was obtained by C. Delaunay; see [De].

In the toric case, there is a notion of a canonical real structure. This is a real structure which is usually defined as complex conjugation on the open $T$-orbit, but we prefer a slightly different and more general definition. Let $\sigma: T \rightarrow T$ be the involutive anti-holomorphic automorphism of the real Lie group $T$ which coincides with inversion on the maximal compact torus $T_{c} \subset T$. If $T \simeq\left(\mathbb{C}^{*}\right)^{n}$ then the real form defined by $\sigma$ is split, i.e., isomorphic to $\left(\mathbb{R}^{*}\right)^{n}$. A canonical real structure on a toric variety $X$ is a real structure which satisfies

$$
\mu(a \cdot x)=\sigma(a) \cdot \mu(x)
$$

for all $x \in X, a \in T$. Of course, a canonical real structure is uniquely defined by the image of one point in the open orbit and any two canonical real structures are related by $\mu^{\prime}(x)=t \cdot \mu(x)$, where $t \in T$ and $\sigma(t) \cdot t=1$

Our goal is to generalize this notion to varieties acted on by reductive algebraic groups. Let $G$ be a connected reductive algebraic group defined over $\mathbb{C}$. We recall that an algebraic involution $\theta$ of $G$ is called a Weyl involution if $\theta(t)=t^{-1}$ for all $t$ in some algebraic torus $T \subset G$. Such an involution is known to be unique up to conjugation by an inner automorphism. By Cartan Fixed Point Theorem, one can always find a maximal compact subgroup $K \subset G$, such that $\theta(K)=K$. Then the corresponding Cartan involution $\tau$ commutes with $\theta$ and the product $\sigma=\tau \circ \theta=\theta \circ \tau$ is an involutive anti-holomorphic automorphism of $G$ defining the split real form. Assume now that $G$ acts on an algebraic variety $X$. Then a real structure $\mu: X \rightarrow X$ is called canonical if $\mu$ satisfies the above condition ( $*$ ) for all $x \in X, a \in G$. We remark that it suffices to check $(*)$ only for $a \in K$, in which case one can replace $\sigma$ by $\theta$.

The most natural generalization of toric varieties to the case of reductive algebraic groups is the notion of spherical varieties, which we recall in Section 1 . 
Suppose $X$ is affine and non-singular. For $X$ spherical a canonical real structure $\mu: X \rightarrow X$ always exists ([A], Theorem 1.2). However, in the non-spherical case such a structure may not exist even if $X$ is homogeneous ([AP], Proposition 6.3).

In this paper, we study the problem of existence of a canonical real structure for all homogeneous spherical varieties, affine or not affine. We also consider the similar question for some complete spherical varieties, namely the so-called wonderful varieties. The definition of wonderful and strict wonderful varieties is recalled in Section 11.

We start with a finiteness theorem for real structures on wonderful varieties (Theorem 2.4). Then we prove some topological properties of a canonical real structure on a wonderful variety provided such a structure exists (Theorem 3.10). After that we show that a canonical real structure exists and is uniquely defined for homogeneous spherical varieties $G / H$ with $H$ self-normalizing (Theorem 4.12) and for their wonderful completions (Theorem4.13). As an application we show that for a spherical subgroup $H \subset G$, whose normalizer is self-normalizing, there is always an anti-holomorphic involution $\sigma: G \rightarrow G$, defining the split real form and such that $\sigma(H)=H$ (Theorem 4.14). Finally, we give an estimate of the total number of real form orbits in $\mathbb{R} X$ for the canonical real structure on a strict wonderful variety $X$ (Theorem 5.19).

\section{Wonderful VARIETIES}

Recall that $G$ is a connected reductive algebraic group over $\mathbb{C}$. A normal algebraic $G$-variety $X$ is called spherical if $X$ contains an open orbit of a Borel subgroup $B \subset G$. We denote the open orbits of $B$ and $G$ on $X$ by $X_{B}^{\circ}$ and $X_{G}^{\circ}$ respectively.

The following definition is due to D. Luna ([Lu1]). An algebraic $G$-variety $X$ is called wonderful if

(i) $X$ is complete and smooth;

(ii) $X$ admits an open $G$-orbit whose complement consists of a finite union of smooth prime divisors $X_{1} \ldots, X_{r}$ with normal crossings;

(iii) the $G$-orbit closures of $X$ are given by the partial intersections of the $X_{i}$ 's.

Remark that a wonderful variety $X$ has a unique closed $G$-orbit. The latter is the full intersection of the boundary divisors $X_{i}$ of $X$.

D. Luna proved that wonderful $G$-varieties are spherical. The connected center of $G$ acts trivially on a wonderful variety, so if $G$ acts effectively then $G$ is semisimple. If a spherical homogeneous space $G / H$ admits an equivariant wonderful embedding then such an embedding is unique up to a $G$-isomorphism; see [Lu1 and references therein. 
By a theorem of F. Knop, a wondferful equivariant embedding of $G / H$ always exists if the spherical subgroup $H$ is self-normalizing in $G$; see $[\mathrm{K}]$.

Proposition 1.1. Let $X$ be a wonderful variety and let $X_{G}^{\circ}=G / H$. Then $H$ has finite index in its normalizer.

Proof. See Section 4.4 in [Br1].

A wonderful variety is called strict if each of its points has a selfnormalizing stabilizer. The class of strict wonderful varieties includes flag varieties and De Concini-Procesi compactifications ([DP]). Strict wonderful varieties are classified in $[\mathrm{BCF}]$.

For any variety $X$, let $\operatorname{Aut}(X)$ denote the automorphism group of $X$. We will need the following proposition describing the identity component $\operatorname{Aut}_{0}(X)$ for a wonderful $G$-variety $X$.

Proposition 1.2 ([Br2], Theorem 2.4.2). If $X$ is wonderful under $G$ then $\operatorname{Aut}_{0}(X)$ is semisimple and $X$ is wonderful under the action of $\operatorname{Aut}_{0}(X)$.

In addition, we have the following proposition, for which we could not find a reference.

Proposition 1.3. Let $X$ be a wonderful variety. Then $\operatorname{Aut}_{0}(X)$ has finite index in $\operatorname{Aut}(X)$.

Proof. Write $X_{G}^{\circ}=G / H$, where $H$ is the stabilizer of a point $x_{0} \in X_{G}^{\circ}$. Let $N$ be the normalizer of $H$ in $G$. By Proposition 1.1 the orbit $N \cdot x_{0}$ is finite. For any $\alpha \in \operatorname{Aut}(X)$ and $g \in \operatorname{Aut}_{0}(X)$ put

$$
\iota_{\alpha}(g)=\alpha \cdot g \cdot \alpha^{-1} \text {. }
$$

Let $L$ denote the group of all automorphisms of the group $\operatorname{Aut}_{0}(X)$. Then we have the homomorphism

$$
\varphi: \operatorname{Aut}(X) \rightarrow L, \quad \varphi(\alpha)=\iota_{\alpha},
$$

whose image contains the group of inner automorphisms of $\operatorname{Aut}_{0}(X)$. Since the latter group is semisimple by Proposition $1.2, \operatorname{Im}(\varphi)$ has finitely many connected components. We now prove that $\operatorname{Ker}(\varphi)$ is finite. It then follows that $\operatorname{Aut}(X)$ has finitely many connected components.

If $\alpha \in \operatorname{Ker}(\varphi)$ then $\alpha$ commutes with all automorphisms from $G$. Thus $\alpha\left(g x_{0}\right)=g \alpha\left(x_{0}\right)$ for all $g \in G$. Since $X$ has only one open $G$ orbit, we have $\alpha\left(X_{G}^{\circ}\right)=X_{G}^{\circ}$ and, in particular, $\alpha\left(x_{0}\right)=a x_{0}$ for some $a \in G$. Now take $g \in H$. Then

$$
a x_{0}=\alpha\left(x_{0}\right)=\alpha\left(g x_{0}\right)=g \alpha\left(x_{0}\right)=\operatorname{gax}_{0},
$$


ON THE CANONICAL REAL STRUCTURE ON WONDERFUL VARIETIES 5

hence $a g a^{-1} \in H$ and $a \in N$. Since the $N$-orbit of $x_{0}$ is finite, there are only finitely many possibilities for $a x_{0}$. But, for $\alpha\left(x_{0}\right)$ fixed, $\alpha$ is uniquely determined on the open $G$-orbit and thus everywhere on $X$.

\section{Finiteness theorem}

The group $\operatorname{Aut}(X)$ acts on the set of real structures on $X$ by

$$
\mu \mapsto \alpha \cdot \mu \cdot \alpha^{-1}
$$

For $X$ wonderful, we prove that this action has only finitely many orbits.

Theorem 2.4. Let $X$ be a wonderful variety. Then, up to an automorphism of $X$, there are only finitely many real structures on $X$.

Proof. Assume that $X$ has at least one real structure $\mu_{0}$. Then $\operatorname{Aut}(X)$ orbits on the set of real structures on $X$ are in one-to-one correspondence with the cohomology classes from $H^{1}\left(\mathbb{Z}_{2}\right.$, Aut $\left.(X)\right)$, where the generator $\gamma \in \mathbb{Z}_{2}$ acts on $\operatorname{Aut}(X)$ by sending $\alpha$ to $\mu_{0} \alpha \mu_{0}$. We now use the exact cohomology sequence, associated with the normal subgroup $\operatorname{Aut}_{0}(X) \triangleleft \operatorname{Aut}(X)$. From Corollary 3 in I.5.5 of [S] it follows that $H^{1}\left(\mathbb{Z}_{2}, \operatorname{Aut}(X)\right)$ is finite if the following two conditions are fulfilled:

(1) $\operatorname{Aut}(X) / \operatorname{Aut}_{0}(X)$ is finite;

(2) for the $\mathbb{Z}_{2}$-action on $\operatorname{Aut}_{0}(X)$ obtained by twisting the given action by an arbitrary cocycle $a \in Z^{1}\left(\mathbb{Z}_{2}\right.$, Aut $\left.(X)\right)$ the corresponding cohomology set $H^{1}\left(\mathbb{Z}_{2},{ }_{a} \operatorname{Aut}_{0}(X)\right)$ is finite.

We have just proved (1). Since $\operatorname{Aut}_{0}(X)$ is linear algebraic, (2) follows from Borel-Serre's Theorem (see [BS]).

\section{General properties of EQUivariant ReAl STRUCtURES}

Let $X$ be a non-singular complex algebraic variety with a real structure $\mu: X \rightarrow X$. Suppose $G$ is a connected algebraic group acting on $X$ and let $\sigma: G \rightarrow G$ be an involutive anti-holomorphic automorphism of $G$ as a real algebraic group. Then the fixed point subgroup

$$
G^{\sigma}=\{g \in G \mid \sigma(g)=g\}
$$

is real algebraic and its identity component $G_{0}^{\sigma}$ is a closed real Lie subgroup in $G$.

We call $\mu$ a $\sigma$-equivariant real structure if

$$
\mu(g \cdot x)=\sigma(g) \cdot \mu(x) \quad \text { for all } g \in G, x \in X .
$$


Later on, we will be interested in the case when $G^{\sigma}$ is a split real form of a reductive group $G$; see Introduction. However, in the following elementary lemma $G$ and $\sigma$ are arbitrary.

Lemma 3.5. Let $H \subset G$ be an algebraic subgroup and let $X=G / H$. Suppose $x_{0} \in \mathbb{R} X$. Then the connected component of $\mathbb{R} X$ through $x_{0}$ coincides with $G_{0}^{\sigma} \cdot x_{0}$. The orbit $G_{0}^{\sigma} \cdot x_{0}$ is Zariski dense in $X$.

Proof. Let $n$ be the complex dimension of $X$. Then the real dimension of $\mathbb{R} X$ is also $n$. Since $\mu$ is $\sigma$-equivariant, we have $G^{\sigma}\left(x_{0}\right) \subset \mathbb{R} X$. Thus it suffices to show that $\operatorname{dim} G_{0}^{\sigma}\left(x_{0}\right) \geq n$. Let $G_{x_{0}}$ be the stabilizer of $x_{0}$ in $G$. Then $G_{0}^{\sigma} \cap G_{x_{0}}$ is a totally real submanifold in $G_{x_{0}}$, hence

$$
\operatorname{dim}_{\mathbb{R}} G_{0}^{\sigma} \cap G_{x_{0}} \leq \operatorname{dim}_{\mathbb{C}} G_{x_{0}},
$$

and so we obtain

$$
\operatorname{dim} G_{0}^{\sigma} \cdot x_{0}=\operatorname{dim} G_{0}^{\sigma}-\operatorname{dim} G_{0}^{\sigma} \cap G_{x_{0}} \geq \operatorname{dim}_{\mathbb{C}} G-\operatorname{dim}_{\mathbb{C}} G_{x_{0}}=n .
$$

Finally, since $G_{0}^{\sigma} \cdot x_{0} \subset X$ is a totally real submanifold of maximal possible dimension, $G_{0}^{\sigma} \cdot x_{0}$ is not contained in an algebraic subvariety of dimension smaller than $n$.

From now on $G$ is reductive. We need some preparatory lemmas on the involution $\sigma: G \rightarrow G$ defining the split real form of $G$. We also fix some notation, which will be used all the time in the sequel. So let $T \subset G$ be a torus, on which $\sigma$ acts as the involutive anti-holomorphic automorphism with fixed point subgroup being the non-compact real part of $T$. In coordinates, if $T \simeq\left(\mathbb{C}^{*}\right)^{r}$ then

$$
\sigma\left(z_{1}, \ldots, z_{r}\right)=\left(\bar{z}_{1}, \ldots, \bar{z}_{r}\right), \quad z=\left(z_{1}, \ldots, z_{r}\right) \in\left(\mathbb{C}^{*}\right)^{r}
$$

Lemma 3.6. Let $\chi$ be a character of $T$. Then $\overline{\chi \circ \sigma}=\chi$.

Proof. Take $t$ in the non-compact real part of $T$. Then $\sigma(t)=t$ and the value of $\chi$ is real. This shows that the weights $\overline{\chi \circ \sigma}$ and $\chi$ coincide on real points, hence also everywhere by analytic extension.

Lemma 3.7. Let $\mathfrak{g}$ be the Lie algebra of $G$. Denote the associated involution of $\mathfrak{g}$ again by $\sigma$. Then all root spaces in $\mathfrak{g}$ are $\sigma$-stable.

Proof. Let $\alpha: T \rightarrow \mathbb{C}^{*}$ be a root, $\mathfrak{g}_{\alpha}$ the corresponding root space, $X_{\alpha} \in \mathfrak{g}_{\alpha}$, and $t \in T$. Then

$$
\operatorname{Ad}(t) \cdot X_{\alpha}=\alpha(t) X_{\alpha}
$$

implies

$$
\operatorname{Ad}(\sigma(t)) \cdot \sigma\left(X_{\alpha}\right)=\overline{\alpha(t)} \sigma\left(X_{\alpha}\right)
$$


or, equivalently,

$$
\operatorname{Ad}(t) \cdot \sigma\left(X_{\alpha}\right)=\overline{\alpha \circ \sigma(t)} \sigma\left(X_{\alpha}\right)=\alpha(t) \sigma\left(X_{\alpha}\right)
$$

where the last equality follows from Lemma 3.6. Therefore $\sigma\left(X_{\alpha}\right) \in \mathfrak{g}_{\alpha}$, showing that the root spaces are $\sigma$-stable.

Corollary 3.8. With the above choice of $T$ we have $\sigma(B)=B$ and $\sigma(P)=P$ for any Borel subgroup $B \subset G$ containing $T$ and any parabolic subgroup $P \subset G$ containing $B$.

Proof. The Lie algebras of $\mathfrak{p}$ and $\mathfrak{b}$ are spanned by root spaces and the Lie algebra $\mathfrak{t}$ of $T$, so Lemma 3.7 applies.

We will assume throughout the paper that $T, B$ and $P$ are chosen as in Corollary 3.8.

Proposition 3.9. With the above choice of $P$, define a self-map of the flag variety $X=G / P$ by $\mu(g \cdot P)=\sigma(g) \cdot P$. Then $\mu$ is a $\sigma$-equivariant real structure on $X$. Such a structure is uniquely defined. The set $\mathbb{R} X$ is the unique closed $G_{0}^{\sigma}$-orbit on $X$. In particular, the (possibly disconnected) real form $G^{\sigma}$ is transitive on $\mathbb{R} X$.

Proof. Clearly, the map $\mu$ is correctly defined, anti-holomorphic, $\sigma$ equivariant, and involutive. If there is another $\sigma$-equivariant real structure $\mu^{\prime}$ on $X$, then the product $\mu^{\prime} \cdot \mu$ is an automorphism of $X$ commuting with the $G$-action. Since $P$ is self-normalizing, such an automorphism is the identity map, hence $\mu^{\prime}=\mu$. By construction, the base point $e \cdot P$ is contained in $\mathbb{R} X$. According to Lemma 3.5, each connected component of $\mathbb{R} X$ is a closed $G_{0}^{\sigma}$-orbit. By [W] such an orbit is unique, so $\mathbb{R} X$ is connected and coincides with that orbit. The last assertion is now obvious.

For a wonderful variety $X$, the existence of a $\sigma$-equivariant real structure requires some work involving Luna-Vust invariants of spherical homogeneous spaces. We postpone this until the next section. Here, assuming that such a structure $\mu$ exists, we study geometric properties of $\mathbb{R} X$. The notation is as in Section 1. In particular,

$$
Y=X_{1} \cap \ldots \cap X_{r}
$$

is the unique closed $G$-orbit in $X$. Note that $\mu(Y)=Y$ and $\mathbb{R} X \cap Y$ is the unique closed $G_{0}^{\sigma}$-orbit in $Y$ by Proposition 3.9 .

Theorem 3.10. Let $X$ be any wonderful G-variety equipped with a $\sigma$-equivariant real structure $\mu$. Then:

(i) $G_{0}^{\sigma}$ has finitely many orbits on $\mathbb{R} X$;

(ii) $\mathbb{R} X \cap G x \neq \emptyset$ for any $x \in X$; 
(iii) there is exactly one closed $G_{0}^{\sigma}$-orbit in $\mathbb{R} X$; this orbit is contained in the closed $G$-orbit and is $G^{\sigma}$-homogeneous;

(iv) $\mathbb{R} X$ is connected.

Proof. (i) $\mathbb{R} X$ is a non-empty real algebraic set. In particular, $\mathbb{R} X$ has finitely many connected components. By Lemma 3.5, each of them is one $G_{0}^{\sigma}$-orbit.

(ii) We choose $B$ as in Corollary 3.8. We prove first that $\mu$ preserves $G$-orbits. This is clear for the open orbit, because its $\mu$-image is also an open orbit which is unique. Since the orbit structure is well understood (see Section 1), it is enough to prove that $\mu\left(X_{i}\right)=X_{i}$, where $X_{i}$ are the boundary divisors. Equivalently, it suffices to prove that the $G$ invariant valuation $v_{i}$ centered on $X_{i}$ is $\mu$-invariant in the sense that

$$
v_{i}(\overline{f \circ \mu})=v_{i}(f)
$$

for any $f \in \mathbb{C}(X) \backslash\{0\}$. It is enough to check this on $B$-eigenfunctions (see Appendix A.1), but then Lemma 3.6 yields the required equality.

Now, let $G \cdot x$ be any $G$-orbit on $X$ and let $\operatorname{cl}(G \cdot x)$ be its Zariski closure within $X$. Since $G \cdot x$ is $\mu$-stable, so is $\operatorname{cl}(G \cdot x)$. Note that $Y \subset \operatorname{cl}(G \cdot x)$, therefore $Z:=\mathbb{R} X \cap \operatorname{cl}(G \cdot x) \neq \emptyset$. Furthermore, $\operatorname{cl}(G \cdot x)$ is a non-singular variety and $Z \subset \operatorname{cl}(G \cdot x)$ is a totally real submanifold of maximal possible dimension. Therefore $Z$ is not contained in the boundary $\operatorname{cl}(G \cdot x) \backslash G \cdot x$.

(iii) Given a closed orbit $G_{0}^{\sigma} \cdot y \subset \mathbb{R} X$, consider the orbit $G^{\sigma} \cdot y$, which is also closed, and take a fixed point of the real form $B^{\sigma} \subset B$ thereon. The existence of such a point follows from Borel's theorem for connected split solvable groups. Assuming $B^{\sigma} \cdot y=y$, we also have $B \cdot y=y$. But then $G \cdot y$ is projective, i.e., $G \cdot y=Y$. Thus our statement is reduced to the case of flag varieties, and we can apply Proposition 3.9

(iv) Assume $\mathbb{R} X$ is disconnected. Since $\mathbb{R} X \cap Y$ is connected, we can find a connected component $W$ of $\mathbb{R} X$, such that $W \cap Y=\emptyset$. Then $W$ is a closed $G_{0}^{\sigma}$-orbit and $G^{\sigma} \cdot W$ is a closed $G^{\sigma}$-orbit, which also has empty intersection with $Y$. On the other hand, by the above argument, $B^{\sigma}$ has a fixed point on $G^{\sigma} \cdot W$. Since that fixed point is also fixed by $B$, it belongs to the closed $G$-orbit $Y$. We get a contradiction showing that $\mathbb{R} X$ is in fact connected.

\section{The CANONicAl ReAl Structure}

Recall that $T$ and $B$ are chosen as in Corollary 3.8 . 
Proposition 4.11. Any spherical subgroup $H \subset G$ is conjugate to $\sigma(H)$ by an inner automorphism of $G$.

Proof. We note first that $\sigma(H)$ is a spherical subgroup of $G$. We shall prove that the Luna-Vust invariants attached to $X_{1}=G / H$ and $X_{2}=$ $G / \sigma(H)$ are the same and then use the theorem in Appendix A.1, from where we also take the notations.

Consider the map $\mu: X_{1} \rightarrow X_{2}$ defined by

$$
X_{1} \ni g \cdot H \stackrel{\mu}{\mapsto} \sigma(g) \cdot \sigma(H) \in X_{2} .
$$

We show that $\mu$ defines a bijection between the sets of $B$-eigenfunctions on $X_{2}$ and $X_{1}$. Moreover, the associated map $\Lambda^{+}\left(X_{2}\right) \longrightarrow \Lambda^{+}\left(X_{1}\right)$ is the identity map. Namely, let $f$ be a $B$-eigenfunction in $\mathbb{C}\left(X_{2}\right)$ and let $\lambda$ be its $B$-weight. Then the complex conjugate of $f \circ \mu$ is a $B$ eigenfunction in $\mathbb{C}\left(X_{1}\right)$ with weight $\overline{\lambda \circ \sigma}$. The latter is equal to $\lambda$ by Lemma 3.6. Since we can apply the same argument to the map $\mu^{-1}$, it follows that the weight lattices of $X_{1}$ and $X_{2}$ coincide and $\mu$ induces the identity map on $\Lambda^{+}\left(X_{2}\right)=\Lambda^{+}\left(X_{1}\right)$.

Further, consider the map $\mathcal{V}\left(X_{1}\right) \rightarrow \mathcal{V}\left(X_{2}\right)$, defined by

$$
v \mapsto(f \mapsto v(\overline{f \circ \mu})) .
$$

This map is obviously bijective. Namely, its inverse is defined analogously by means of the mapping $\mu^{-1}: X_{2} \rightarrow X_{1}$.

Finally, there is a natural bijection $\iota: \mathcal{D}_{G, X_{1}} \rightarrow \mathcal{D}_{G, X_{2}}$ sending $D$ to $\pi_{2}\left[\sigma\left(\pi_{1}^{-1}(D)\right]\right.$ where $\pi_{1}$ and $\pi_{2}$ are the projections from $G$ to $X_{1}$ and $X_{2}$ respectively. For this mapping, $\varphi_{\iota(D)}$ evaluated on $\overline{\lambda \circ \sigma}$ gives the same result as $\varphi_{D}$ evaluated on $\lambda$. By Lemma 3.6, $\overline{\lambda \circ \sigma}$ coincides with $\lambda$, and so we have $\varphi_{\iota(D)}=\varphi_{D}$. Similarly, $G_{\iota(D)}=\sigma\left(G_{D}\right)=G_{D}$ because $G_{D}$ is a parabolic subgroup containing $B$.

Theorem 4.12. Let $H$ be a spherical subgroup of $G$ and $a \in G$ such that $\sigma(H)=a H a^{-1}$. The assignment

$$
\mu_{0}: g H \mapsto \sigma(g) a H
$$

defines an anti-holomorphic $\sigma$-equivariant diffeomorphism of $G / H$. If $H$ is self-normalizing then this map is involutive, hence a $\sigma$-equivariant real structure on $G / H$. Furthermore, for $H$ self-normalizing a $\sigma$ equivariant real structure on $G / H$ is uniquely defined.

Proof. The first assertion follows from Proposition 4.11. Further, since $\sigma$ is an involution of $G, \sigma(a) a$ belongs to the normalizer of $H$ in $G$. The latter coincides with $H$. This proves the second assertion. The product of two $\sigma$-equivariant real structures on $G / H$ is an automorphism of 
$G / H$ commuting with the $G$-action. For $H$ self-normalizing in $G$ such an automorphism is the identity map, and the last assertion follows.

Theorem 4.13. Let $H$ be a self-normalizing spherical subgroup of $G$ and let $X$ be the wonderful completion of $G / H$. Then there exists one and only one $\sigma$-equivariant real structure of $X$.

Proof. Let $\iota: G / H \rightarrow X$ be the given wonderful completion and let $\bar{\iota}: G / H \rightarrow \bar{X}$ be the corresponding anti-holomorphic map with $\bar{X}$ being the complex conjugate of $X$. Recall that $\bar{X}=X$ as sets and that the sheafs of regular functions of $\bar{X}$ and $X$ are complex conjugate.

We endow $\bar{X}$ with the $G$-action $(g, x) \mapsto \sigma(g) \cdot x$, where $(g, x) \mapsto g \cdot x$ is the given action of $G$ on $X$. Note that this new action is regular on $\bar{X}$.

Consider the real structure $\mu_{0}$ introduced in Theorem 4.12, Then $\bar{\iota} \circ \mu_{0}$ is again a wonderful completion of $G / H$. Since two wonderful completions of $G / H$ are $G$-isomorphic, there exists a $G$-isomorphism $\mu: X \rightarrow \bar{X}$ such that $\mu \circ \iota=\bar{\iota} \circ \mu_{0}$. The map $\mu$ defines a $\sigma$-equivariant real structure on $X$.

Finally, a $\sigma$-equivariant real structure on $X$ is defined by its restriction to the open $G$-orbit in $X$. The restriction is unique by Theorem 4.12 ,

In the remainder, the real structure defined in Theorem 4.13 is called the canonical real structure of $X$. We want to give here a grouptheoretical application of Theorem 4.13.

Theorem 4.14. If $H \subset G$ is a spherical subgroup with self-normalizing normalizer then there exists an anti-holomorphic involution $\sigma: G \rightarrow G$, defining the split real form and such that $\sigma(H)=H$. Moreover, one can find a Borel subgroup $B \subset G$, such that $B \cdot H$ is open in $G$ and $\sigma(B)=B$.

Proof. Let $N$ be the normalizer of $H$ in $G$. We start with some $\sigma$ and take $a \in G$ as in Theorem 4.12, i.e., $\sigma(H)=a H a^{-1}$. Then, of course, $\sigma(N)=a N a^{-1}$. Let $X$ be a wonderful equivariant completion of $G / N$ and let $\mu$ be the canonical $\sigma$-equivariant real structure on $X$. By Theorem 3.10 we can find a $\mu$-fixed point in the open orbit. Let $\mu\left(g_{0} \cdot N\right)=g_{0} \cdot N$. Replace $\sigma$ by $\sigma_{1}=i_{g_{0}}^{-1} \sigma i_{g_{0}}$, where $i_{g_{0}}$ is the inner automorphism of $G$ given by $x \mapsto g_{0} x g_{0}^{-1}$. Also, replace $\mu$ by $\mu_{1}=$ $g_{0}^{-1} \mu g_{0}$. A straightforward calculation shows that $\mu_{1}(g x)=\sigma_{1}(g) \mu_{1}(x)$ for all $g \in G, x \in X$, i.e., $\mu_{1}$ is a $\sigma_{1}$-equivariant real structure on $X$. Moreover, for the new pair $\left(\mu_{1}, \sigma_{1}\right)$ we have

$$
\mu_{1}(e \cdot N)=\left(g_{0}^{-1} \mu g_{0}\right)(e \cdot N)=g_{0}^{-1} \mu\left(g_{0} \cdot N\right)=e \cdot N .
$$


Comparing the stabilizers at $e \cdot N$ and $\mu_{1}(e \cdot N)$, we get

$$
\sigma_{1}(N)=N \text {. }
$$

It follows that

$$
N=\sigma_{1}(N)=i_{g_{0}}^{-1} \sigma i_{g_{0}}(N)=g_{0}^{-1} \sigma\left(g_{0}\right) \sigma(N) \sigma\left(g_{0}\right)^{-1} g_{0} .
$$

As we have seen, $\sigma(N)=a N a^{-1}$. Substituting this in the previous equality, we get $g_{0}^{-1} \sigma\left(g_{0}\right) a \in N$, and it follows that $\sigma_{1}(H)=H$.

Now, assuming $\sigma(H)=H$ consider the subset $\Omega \subset G / B$ whose points correspond to the Borel subgroups $B_{*} \subset G$ with $B_{*} \cdot H$ open in $G$. Then $\Omega$ is Zariski open and $\sigma$-stable. The subset of $\sigma$-fixed Borel subgroups is a totally real submanifold in $G / B$, having maximal possible dimension. Thus its intersection with $\Omega$ is non-empty.

Remark 4.15. The normalizer of a spherical subgroup is in general not self-normalizing, see Example 4 in $\mathrm{Av}$. In Theorem 4.14, we do not know if the condition of $N$ being self-normalizing is essential.

\section{ReAl PART: LOCAL STRUCTURE AND $G_{0}^{\sigma}$-ORBitS}

Let $X$ be a strict wonderful $G$-variety of rank $r$ equipped with the canonical real structure $\mu$. For a complex vector space $V$ and an antilinear map $\nu: V \rightarrow V$ we denote by the same letter $\nu$ the induced anti-holomorphic map of $\mathbb{P}(V)$.

Proposition 5.16. There exist a simple $G$-module $V$ with the associated representation $\rho: G \rightarrow \mathrm{GL}(V)$, an anti-linear involutive map $\nu: V \rightarrow V$, and an embedding $\varphi: X \rightarrow \mathbb{P}(V)$, such that

(i) $\nu(\rho(g) \cdot v)=\rho(\sigma(g)) \cdot \nu(v) \quad(v \in V)$,

(ii) $\varphi(g x)=\rho(g) \cdot \varphi(x) \quad(x \in X)$ and

(iii) $\varphi(\mu x)=\nu \varphi(x) \quad(x \in X)$.

In particular, $\mathbb{R} X$ is $G^{\sigma}$-equivariantly embedded into the real projective space $\mathbb{R P}(V) \subset \mathbb{P}(V)$, defined by $\nu$.

Proof. Since $X$ is a non-singular projective $G$-variety, $X$ can be $G$ equivariantly embedded into the projectivization of a $G$-module. Let $\varphi: X \rightarrow \mathbb{P}(V)$ be such an embedding and let $\rho: G \rightarrow \mathrm{GL}(V)$ denote the representation associated to $V$. Since $X$ is a strict wonderful variety, we may choose $V$ to be simple; see $[\mathrm{P}]$.

Now, equip the complex conjugate vector space $\bar{V}$ with the $G$-module structure given by $g \mapsto \overline{\rho(\sigma(g))}$. By Lemma 3.6, it follows that the $G$-modules $V$ and $\bar{V}$ are isomorphic. In other words, we have an antilinear map $\nu: V \rightarrow V$ satisfying (i). Though $\nu$ is not necessarily involutive, we can modify $\nu$ to get this property. As in Appendix A.2. 
let $v^{-}$be a lowest weight vector of $V$. Then $\nu\left(v^{-}\right)$is also a lowest weight vector, hence $\nu\left(v^{-}\right)=c v^{-}$for some $c \in \mathbb{C}^{*}$. This implies $\nu^{2}\left(v^{-}\right)=\nu\left(c v^{-}\right)=\bar{c} \cdot \nu\left(v^{-}\right)=|c|^{2} v^{-}$. Replacing $\nu$ by $\nu /|c|$, we get an involutive anti-linear map satisfying (i).

Since (ii) is clear from the construction, it remains to show (iii). Note that $\nu \circ \varphi \circ \mu$ is another $G$-equivariant embedding of $X$ into $\mathbb{P}(V)$. Thus (iii) follows from the uniqueness of such an embedding; see $[\mathrm{P}]$.

Let $Z$ be the slice defined in Appendix A.2. We show that $Z$ can be chosen to be $\mu$-stable. As we have seen in Proposition 5.16, the line $\mathbb{C} \cdot v^{-}$is $\nu$-stable. So we may assume that $\nu\left(v^{-}\right)=v^{-}$. Then the tangent space $W:=T_{v^{-}} G \cdot v^{-}$is also $\nu$-stable. Consider the real vector space $\mathbb{R} V=\{v \in V \mid \nu(v)=v\}$ and let $\mathbb{R} W=W \cap \mathbb{R} V$. Then $\mathbb{R} W$ is stable under $L^{\sigma}$ and, also, under the Lie algebra $\mathfrak{l}^{\sigma}$ of $L^{\sigma}$. Now, the center of $\mathfrak{l}^{\sigma}$ is contained in the center of the complexified algebra $\mathfrak{l}=\mathfrak{l}^{\sigma} \otimes \mathbb{C}$ and is therefore represented by semisimple endomorphisms of $\mathbb{R} V$. The complete reducibility theorem for reductive Lie algebras over $\mathbb{R}$ implies that $\mathbb{R} W$ has a $\mathfrak{l}^{\sigma}$-stable complement in $\mathbb{R} V$; see $[\mathrm{C}]$, Ch.IV, $\S$ 4. Call this complement $E_{\mathbb{R}}$. The complexification $E_{\mathbb{R}} \otimes \mathbb{C} \subset V$ is $\mathfrak{l}$-stable and therefore $L$-stable. So we can take $E=E_{\mathbb{R}} \otimes \mathbb{C}$. Note that $E_{\mathbb{R}}=E \cap \mathbb{R} V$ is not just $\mathfrak{l}^{\sigma}$-stable, but also $L^{\sigma}$-stable even if $L^{\sigma}$ is disconnected.

Obviously, $\nu(E)=E$. Furthermore, the linear form $\eta$ in Appendix A.2 can be chosen real. Therefore, using (iii) of Proposition 5.16, we see that $\mu(Z)=Z$. Note that $P^{u} \cdot Z$ is $\mu$-stable and $\mathbb{R}\left(P^{u} \cdot Z\right)=$ $\left(P^{u}\right)^{\sigma} \cdot \mathbb{R} Z$.

The first assertion of the following proposition is a real analogue of Local Structure Theorem in [BLV]; see also Appendix A.2.

Proposition 5.17. (i) The natural mapping

$$
\left(P^{u}\right)^{\sigma} \times(\mathbb{R} Z) \rightarrow\left(P^{u}\right)^{\sigma} \cdot \mathbb{R} Z=\mathbb{R}\left(P^{u} \cdot Z\right)
$$

is a $\left(P^{u}\right)^{\sigma}$-equivariant isomorphism.

(ii) Each $G_{0}^{\sigma}$-orbit in $\mathbb{R} X$ contains points of the slice $Z$.

Proof. The first assertion follows readily from Local Structure Theorem and the above construction of $Z$.

To prove (ii), take a point $x \in \mathbb{R} X$. Since $X$ is wonderful, the orbit $G \cdot x$ is not contained in a prime divisor $D \in \mathcal{D}(X)$. The intersection $G \cdot x \cap\left(\cup_{\mathcal{D}(X)} D\right)$ is a proper Zariski closed subset in $G \cdot x$. By the last assertion of Lemma 3.5, this subset does not contain $G_{0}^{\sigma} \cdot x$. Thus $G_{0}^{\sigma} \cdot x \cap X \backslash \cup_{\mathcal{D}(X)} D \neq \emptyset$, and (ii) follows from (i). 
In the remainder, $x$ denotes a real point in $X_{G}^{\circ} \cap Z$ and $H \subset G$ is the stabilizer of $x$. We assume that $\sigma(B)=B$ and $\mu(Z)=Z$. It follows that $\sigma(H)=H$. Note also that $B \cdot H$ is open in $G$ because the orbit $B \cdot x$ is open in $X$.

As we recall in Appendix A.2, $T$ acts linearly on $Z$ and the corresponding characters, say $\gamma_{1}, \ldots, \gamma_{r}$, are linearly independent. These characters are usually called spherical roots of $X$. Further, we have

$$
T \cap H=\bigcap_{i} \operatorname{ker} \gamma_{i}
$$

Set

$$
A=T / T \cap H
$$

and let ${ }_{2} A \subset A$ be the subgroup of elements of order at most 2 . Note that any element $t \in T$ can be uniquely written as

$$
t=t_{0} t_{1}, \quad \text { where } t_{0} \in T_{0}^{\sigma} \text { and } \sigma\left(t_{1}\right)=t_{1}^{-1} .
$$

Such a decomposition of $t$ will be referred to as the decomposition of $t$ with respect to $\sigma$.

Proposition 5.18. The $T_{0}^{\sigma}$-orbits of $\mathbb{R} Z \cap X_{G}^{\circ}$ are in one-to-one correspondence with the elements of ${ }_{2} A$. In particular, the number of such orbits does not exceed $2^{r}$.

Proof. Let $t \in T$ and let $y=t \cdot x$ be a real point. Then $\left(\gamma_{i} \circ \sigma\right)(t)=\gamma_{i}(t)$ for every spherical root $\gamma_{i}$ of $X$. By Lemma 3.6, it follows that $\gamma_{i}(t)$ is real-valued. If $t=t_{0} t_{1}$ is the decomposition of $t$ with respect to $\sigma$, then we have $\gamma_{i}\left(t_{1}\right)= \pm 1$. Therefore $t_{1}^{2} \in H$. Assigning to $y \in \mathbb{R} Z \cap X_{G}^{\circ}$ the image of $t_{1}$ in $A$, we get a correctly defined map from the set of $T_{0}^{\sigma}$-orbits on $\mathbb{R} Z \cap X_{G}^{\circ}$ to ${ }_{2} A$ :

$$
\alpha: T_{0}^{\sigma} \backslash\left(\mathbb{R} Z \cap X_{G}^{\circ}\right) \rightarrow{ }_{2} A
$$

The injectivity of $\alpha$ is obvious. To prove the surjectivity, take any $t \in T$, such that $t^{2} \in H$. Then $\gamma_{i}\left(t^{2}\right)=1$, hence $\gamma_{i}(t)= \pm 1$. It follows that $t \cdot x$ is a real point. Furthermore, $\gamma_{i}\left(t_{0}\right)=1$ and $\gamma_{i}\left(t_{1}\right)=\gamma_{i}(t)$. Hence $t \cdot H=t_{1} \cdot H$ and $\alpha\left(T_{0}^{\sigma} \cdot x\right)=t \bmod T \cap H$.

Let $I$ denote a subset of $\{1, \ldots, r\}$ and let $O_{I} \subset X$ be the corresponding $G$-orbit. Recall that $O_{I}$ is $\mu$-stable.

Theorem 5.19. (i) Each $G_{0}^{\sigma}$-orbit in $\mathbb{R} O_{I}$ intersects the slice $Z$ in a finite number of $T_{0}^{\sigma}$-orbits. The number of $T_{0}^{\sigma}$-orbits in $\mathbb{R} Z \cap O_{I}$ does not exceed $2^{r-|I|}$.

(ii) $\mathbb{R} O_{I}$ contains at most $2^{r-|I|} G^{\sigma}$-orbits. 
(iii) The total number of $G_{0}^{\sigma}$-orbits in $\mathbb{R} X$ is smaller than or equal to

$$
\sum_{k=0}^{r} 2^{k}\left(\begin{array}{l}
r \\
k
\end{array}\right) .
$$

Proof. Recall that the $G$-orbit closures in $X$ are also strict wonderful $G$-varieties. Furthermore, the rank of the orbit closure cl $\left(O_{I}\right)$ equals $r-$ $|I|$; see Sect. 3.2 in [Lu2]. Thus (i) follows from (ii) of Proposition 5.17, along with the estimate in Proposition 5.18. From (i) we get (ii), and (iii) is obtained by summing up over all $G$-orbits.

Example 1. Let $\left(\mathbb{P}^{n}\right)^{*}$ denote the variety of hyperplanes of $\mathbb{C}^{n+1}$ and let $X=\mathbb{P}^{n} \times\left(\mathbb{P}^{n}\right)^{*}$ be acted on diagonally by $G=P G L_{n+1}(\mathbb{C})$. Suppose $n>1$. Then $X$ is a strict wonderful variety of rank 1 . The canonical real structure $\mu$ is defined by the complex conjugation on each factor of $X$. Moreover, $G_{0}^{\sigma}=G^{\sigma}=P G L_{n+1}(\mathbb{R})$ acts on $\mathbb{R} X$ with two orbits.

Example 2. Consider the quadratic form

$$
F(z)=z_{1}^{2}+\ldots+z_{p}^{2}-z_{p+1}^{2}-\ldots-z_{p+q}^{2}, \quad q \geq p>0, \quad p+q>2 .
$$

The corresponding orthogonal group $G=S O_{F}$ acts on $X=\mathbb{P}^{m}$ as a subgroup of $\mathrm{SL}_{m+1}(\mathbb{C})$, where $m=p+q-1$. Under this action, $X$ is a two-orbit $G$-variety. The closed $G$-orbit is given by the equation $F=0$. Again, $X$ is a strict wonderful variety of rank 1. Let $\mu: X \rightarrow X$ and $\sigma: G \rightarrow G$ be the involutive mappings defined by complex conjugation. Then $\mu$ is a $\sigma$-equivariant real structure on $X$. Note that $\sigma$ defines a split real form of $G$ only for $q=p$ or $q=p+1$. The real part $\mathbb{R} X$ is the real projective space $\mathbb{R P}^{m}$, on which $G_{0}^{\sigma}$ acts with three orbits: $F>0, F<0$ and $F=0$.

Remark 5.20. Starting with a real semisimple symmetric space, A. Borel and L. Ji considered the wonderful completion of the complexified homogeneous space. In this special setting, the completion is defined over $\mathbb{R}$ in a natural way. For the description of real group orbits on the set of its real points see [BJ], chapters $5-7$.

\section{ApPEndix A. SPHERICAL VARIETIES: InVARIANTS AND LOCAL STRUCTURE}

\section{A.1. Luna-Vust invariants of spherical homogeneous spaces.} We recollect the definition of the combinatorial invariants attached to a given spherical $G$-variety $X$; see [LV].

Let $\mathbb{C}(X)$ denote the function field of $X$. Then the natural left action of $G$ on $X$ yields a $G$-module structure on $\mathbb{C}(X)$. The weight lattice 
$\Lambda^{+}(X)$ is the set of $B$-weights of the $B$-eigenfunctions of $\mathbb{C}(X)$. Since $X$ is spherical, the $\chi$-weight space of $\mathbb{C}(X)$ is of dimension 1 for every $\chi \in \Lambda^{+}(X)$.

Let $\mathcal{V}(X)$ be the set of $G$-invariant discrete $\mathbb{Q}$-valued valuations of $\mathbb{C}(X)$. Consider the mapping

$$
\rho: \mathcal{V}(X) \rightarrow \operatorname{Hom}\left(\Lambda^{+}(X), \mathbb{Q}\right), \quad v \mapsto\left(\chi \mapsto v\left(f_{\chi}\right)\right)
$$

where $f_{\chi}$ is a $B$-eigenfunction of $\mathbb{C}(X)$ of weight $\chi$. The map $\rho$ is injective, hence one may regard $\mathcal{V}(X)$ in $\operatorname{Hom}\left(\Lambda^{+}(X), \mathbb{Q}\right)$. Further, this cone is convex and simplicial. The cone $\mathcal{V}(X)$ is called the valuation cone of $X$; see for instance $[\mathrm{Br} 1$.

Define the set of colors $\mathcal{D}(X)$ of $X$ as the set of $B$-stable, but not $G$-stable prime divisors of $X$. This is a finite set equipped with two maps, namely, $D \mapsto \rho\left(v_{D}\right)$ and $D \mapsto G_{D}$ with $v_{D}$ (resp. $G_{D}$ ) being the valuation defined by (resp. the stabilizer in $G$ of) the color $D$.

The Luna-Vust invariants of $X$ are given by the triple $\Lambda^{+}(X), \mathcal{V}(X)$, $\mathcal{D}(X)$. For two spherical $G$-varieties $X$ and $X^{\prime}$, the equality $\mathcal{D}(X)=$ $\mathcal{D}\left(X^{\prime}\right)$ means that there exists a bijection $\iota: \mathcal{D}(X) \rightarrow \mathcal{D}\left(X^{\prime}\right)$, such that $G_{D}=G_{\iota(D)}$ and $\rho\left(v_{D}\right)=\rho\left(v_{\iota(D)}\right)$.

Theorem 1.21 ([Lo]). Let $H$ and $H^{\prime}$ be spherical subgroups of $G$. If $H$ and $H^{\prime}$ have the same Luna-Vust invariants then they are $G$-conjugate.

A.2. Local structure. We recall the so-called Local Structure Theorem with special emphasis on the case of wonderful varieties.

Let $G$ denote a connected reductive algebraic group. Fix a Borel subgroup $B$ of $G$ and a maximal torus $T \subset B$ of $G$.

First, consider any normal and irreducible $G$-variety $X$ and let $Y$ be a complete $G$-orbit of $X$. Let $y \in X$ be fixed by the Borel subgroup $B^{-}$of $G$ opposite to $B$ and containing $T$. Let $P$ denote the parabolic subgroup of $G$ opposite to the stabilizer $G_{y}$ and containing $T$. Then $L=P \cap G_{y}$ is a Levi subgroup of $P$, so that $P=L \cdot P^{u}$, where $P^{u}$ is the unipotent radical of $P$. Theorem $1.4 \mathrm{in}$ [BLV] asserts that there exists an affine $L$-variety $Z$, such that $y \in Z$ and the natural map $P^{u} \times Z \rightarrow P^{u} \cdot Z$ is an isomorphism.

Suppose now that $X$ is spherical and denote the set of colors of $X$ by $\mathcal{D}(X)$; see Appendix A.1. Further, if $Y$ is the unique closed $G$-orbit in $X$ then $P^{u} Z$ is the affine set $X \backslash \cup_{\mathcal{D}(X)} D$ and $Z$ is a spherical $L$ variety. In the case of wonderful varieties Theorem 1.4 in [BLV] can be formulated as follows; see [Lu1], Sect. 1.1, 1.2, and [Br1], Sect.2.2-2.4.

Theorem 1.22. Assume $X$ is a wonderful $G$-variety. There exists an affine L-subvariety $Z$ of $X$ containing $y$ such that 
(i) $P^{u} \times Z \rightarrow X \backslash \cup_{\mathcal{D}(X)} D:(p, z) \mapsto p . z$ is an isomorphism.

(ii) The derived group of $L$ acts trivially on $Z$ and $Z$ intersects each $G$-orbit of $X$ in one single $T$-orbit.

(iii) The variety $Z$ is the affine space of dimension equal to the rank $r$ of $X$ Moreover, $Z$ is acted on linearly by $T$ and the corresponding $r$ characters of $T$ are linearly independent.

Note that (ii) is a consequence of the configuration of the $G$-orbit closures in a wonderful variety. To obtain (iii), remark that $Z$ is smooth since so is $X$ and thereafter apply (ii) together with Luna Slice Theorem.

Let us now recollect how the slice $Z$ is constructed in the case of strict wonderful varieties. One may consult [BLV] for a general treatment. Let

$$
\varphi: X \rightarrow \mathbb{P}(V)
$$

be an embedding of $X$ within the projectivization of a finite dimensional $G$-module $V$. Thanks to [P], we can take $V$ to be simple. Then $y$ regarded in $\mathbb{P}(V)$ can be written as $\left[v^{-}\right]$with $v^{-}$being a $B^{-}$-eigenvector of $V$ (unique up to a scalar). Since $L$ is reductive, there exists an $L$ module submodule $E \subset V$ such that

$$
V=T_{v^{-}} G \cdot v^{-} \oplus E,
$$

where $T_{v^{-}} G \cdot v^{-}$stands for the tangent space of the orbit $G \cdot v^{-}$at the point $v^{-}$. Let $\eta$ be the linear form on $V$ such that $\eta\left(v^{-}\right)=1$ and $\eta$ is a $B$-eigenvector. Let $\mathbb{P}\left(\mathbb{C} v^{-} \oplus E\right)_{\eta}$ be the open set of $\mathbb{P}(V)$ on which $\eta$ does not vanish. Then

$$
Z=\varphi^{-1}\left(\mathbb{P}\left(\mathbb{C} v^{-} \oplus E\right)_{\eta}\right)
$$

\section{REFERENCES}

[A] D. Akhiezer, Spherical Stein manifolds and the Weyl involution, Ann. Inst. Fourier, Grenoble 59, (2009), 3, 1029-1041.

[AP] D. Akhiezer and A. Püttmann, Antiholomorphic involutions of spherical complex spaces, Proc. Amer. Math. Soc. 136, (2008), 5, 1649-1657.

[Av] R. Avdeev, The normalizers of solvable spherical subgroups, arXiv: 1107.5175 .

[BJ] A. Borel and L. Ji, Compactifications of Symmetric and Locally Symmetric Spaces, Birkhäuser, 2005.

[BS] A. Borel and J.-P. Serre, Théorèmes de finitude et cohomologie galoisienne, Comment. Math. Helv. 39 (1964), 111-164.

$[\mathrm{BCF}]$ P. Bravi and S. Cupit-Foutou, Classification of strict wonderful varieties, Ann. Inst. Fourier, Grenoble 560, (2010), 2, 641-681.

[Br1] M. Brion, Variétés sphériques, Notes de la session de la S. M. F. "Opérations hamiltoniennes et opérations de groupes algébriques", Grenoble, 1997, 1-60. 
ON THE CANONICAL REAL STRUCTURE ON WONDERFUL VARIETIES 17

[Br2] M. Brion, The total coordinate ring of a wonderful variety, J. Algebra 313, (2007), 1, 61-99.

[BLV] M. Brion, D. Luna and T. Vust, Espaces homogènes sphériques, Invent. Math. 84 (1986), 617-632.

[C] C. Chevalley, Théorie des groupes de Lie, Hermann, Paris, 1968.

[DP] C. De Concini and C. Procesi, Complete symmetric varieties, Invariant theory (Montecatini, 1982), Lecture Notes in Math., 996, Springer, Berlin, 1983, 1-44.

[De] C. Delaunay, Real structures on compact toric varieties, Thèse, Université Louis Pasteur, Strasbourg, 2004.

[K] F. Knop, Automorphisms, root systems, and compactifications of homogeneous varieties, J. Amer. Math. Soc.9 (1996), 153-174.

[Lo] I. Losev, Uniqueness property for spherical homogeneous spaces, Duke Math. J. 147 (2009), 2, 315-343.

[Lu1] D. Luna, Toute variété magnifique est sphérique, Transform. Groups 1 (1996), 3, 249-258.

[Lu2] D. Luna, Variété sphériques de type A, Publ. Math. Inst. Hautes Études Sci. 94 (2001), 161-226.

[LV] D. Luna and T. Vust, Plongements d'espaces homogènes, Comment. Math. Helv., 58 (1983), 186-245.

[P] G. Pezzini, Simple immersions of wonderful varieties, Math. Z., 255 (2007), 793-812.

[S] J.-P. Serre, Galois cohomology, Springer-Verlag, Berlin-Heidelberg, 1997.

$[\mathrm{W}] \quad$ J. Wolf, The action of a real semisimple group on a complex flag manifold I. Orbit structure and holomorphic arc components, Bull. AMS, 75 (1969), 1121-1237.

Dmitri Akhiezer, Institute for Information Transmission Problems, B.KARETNY PER. 19, 101447 Moscow, RUSSIA

E-mail address: akhiezer@iitp.ru

Stéphanie Cupit-Foutou, Ruhr-Universität Bochum, NA 4/67, Bochum, GERMANY

E-mail address: stephanie.cupit@rub.de 